\title{
La distribución espacial de la población inmigrante en dos ciudades medias: Alcalá de Henares y León y su relación con los precios de la vivienda ${ }^{1}$
}

\author{
María Jesús GonzÁlez GonZÁlez \\ Departamento de Geografía y Geología \\ Universidad de León \\ mjgong@unileon.es \\ María Luisa DE LÁZARO Y TORRES \\ Departamento de Geografía Humana \\ Universidad Complutense de Madrid \\ mllazaro@ghis.ucm.es
}

Recibido: 7 de mayo de 2011

Enviado a evaluar: 15 de enero de 2012

Aceptado: 9 de julio de 2012

\section{RESUMEN}

La crisis existente en la actualidad ha frenado la tradicional tendencia al incremento en el número de inmigrantes, aunque esto no se ha producido de forma idéntica en todas las ciudades, ni dentro de éstas en todos los barrios. Estudiaremos los casos de dos ciudades medias muy diferentes, León y Alcalá de Henares a partir del censo de 2001 y el padrón de 2009. Detectaremos en qué barrios existe una mayor concentración de inmigrantes y explicaremos el por qué. Analizaremos la relación entre la distribución espacial de la población inmigrante y la de los precios de la vivienda de segunda mano, para estos mismos años, a través de las cifras obtenidas a partir de más de 4000 anuncios inmobiliarios. Contribuiremos así al debate científico sobre la inmigración extranjera en las ciudades medias y su relación con el precio de la vivienda por barrios.

Palabras clave: inmigración, extranjeros, precios vivienda, León, Alcalá de Henares.

\section{Spatial Distribution of the Immigrant Population in two Medium-Sized} Cities: Alcalá de Henares and León and its Relationship to Housing Prices

\begin{abstract}
The number of immigrants in Spain is similar to the European countries with the longest tradition of immigration, this event has occurred in a very short time. The present crisis has slowed the increase in the number of immigrants. This fact has not occurred identically in all cities, nor in every neighbourhood within them. We will exemplify this in two very different medium cities, León and Alcalá de Henares. We will observe where and why in those cities there is a particular concentration of immigrants. We use census data of 2001 and 2009. We will also use the second hand housing prices from around 4000 real
\end{abstract}

${ }^{1}$ El presente trabajo se ha desarrollado a partir de los proyectos de investigación (I+D) BSO200202432 y SEJ2004-07831, del Ministerio de Ciencia y Tecnología. 
estate advertisements analysed for the same dates. Therefore we will contribute to scientific debate on foreign immigration in the cities and its relation with housing prices.

Key words: Immigration, Foreigner, Housing Prices, León, Alcalá de Henares.

\title{
La distribution spatiale de la population immigrée dans deux villes de taille moyenne: Alcalá de Henares y León et sa relation avec les prix des logements
}

\begin{abstract}
RÉSUMÉ
La crise actuelle a ralenti la tendance habituelle à l'augmentation du nombre d'immigrants, bien que ce phénomène ne se soit pas produit de la même façon dans toutes les villes, ni même dans tous les quartiers qui les conforment. Nous nous proposons d'étudier le cas de deux villes moyennes très différentes, León et Alcalá de Henares à partir du recensement de 2001 et 2009. Nous détecterons dans quels quartiers il existe une plus grande concentration d'immigrants et nous en expliquerons les raisons. Nous analyserons le rapport entre la distribution spatiale de la population immigrante et celle du prix des logements d'occasion, au cours de ces mêmes années, à travers des chiffres obtenus à partir de plus de 4000 annonces immobilières. Nous contribuerons ainsi au débat scientifique sur l'immigration étrangère dans les villes moyennes et son rapport avec le prix du logement par quartiers.
\end{abstract}

Mots-clés: immigration, étrangers, prix du logement, León, Alcalá de Henares.

\section{INTRODUCCIÓN}

Desde los años noventa asistimos a un rápido incremento en el flujo de inmigrantes en toda España. Alcalá de Henares y León no constituyen ninguna excepción a este hecho. España se ha convertido en el país europeo que más inmigrantes ha recibido anualmente en los últimos años, tanto en números absolutos como relativos (Bodega y Martín, 2006, 385), si bien somos conscientes de que actualmente se ha frenado, en todos los países europeos, esta afluencia inmigratoria. Este fenómeno inmigratorio ha ofrecido interés por su volumen y dimensiones (Bodega, Cebrián y Martín Lou, 2008), por su localización espacial, que se concentra en lugares concretos, y por el origen de la población que lo integra, hechos que vamos a analizar para los diferentes barrios en las dos ciudades citadas. La unidad espacial tomada como barrio tiene sus límites definidos por los ayuntamientos respectivos.

El movimiento de la población ha sido tradicionalmente uno de los mejores indicadores para conocer la situación económica en la que están los distintos lugares. Hoy, a pesar de la crisis económica, siguen existiendo grandes diferencias entre la renta per cápita de los países de los que proceden los inmigrantes y España. Mientras sigan existiendo diferencias en los salarios y alguna posibilidad de obtener empleo en España, no dejarán de existir los flujos inmigratorios, si bien en los últimos años la crisis económica los ha frenado.

El crecimiento actual del desempleo ha ralentizado la inmigración en España, aunque no ha cesado, lo que se puede constatar tanto en el caso de Alcalá de Henares como en el de León, a pesar de las diferencias entre ambas ciudades. El ser dos ciudades medias de diferentes características demográficas refuerza el interés por estudiar- 
las para analizar los contrastes. El fenómeno inmigratorio en la ciudad de León dista mucho de tener la intensidad que en otras ciudades españolas. Sin embargo, Alcalá de Henares, como localidad cercana a Madrid -ciudad de gran atractivo socioeconómico para las migraciones hasta la crisis actual-, cuenta con un volumen al menos tres veces mayor que el de la ciudad de León para el fenómeno de la inmigración, de hecho es el municipio con más inmigrantes en la Comunidad Autónoma después de la propia capital. Lo que significa que es uno de los municipios españoles con mayor número de inmigrantes.

En este trabajo hay que tener en cuenta dos aspectos o puntos de partida fundamentales: 1 . Se trata de un estudio a una escala espacial inframunicipal de barrios (no de distritos censales) y no de un estudio general o con datos provinciales o nacionales en los que se abunde en el hecho generalizado de que la inmigración ha supuesto una subida y un sostenimiento de los precios de la vivienda, como hacen algunos autores para Suiza (Degen y Fischer, 2009), Canadá (Akbari y Aydede, 2009), Nueva Zelanda (Coleman y Landom-Lane, 2008; Stillman y Mare, 2009) o España (González y Ortega, 2009). 2. En España las fuentes demográficas contabilizan el número de extranjeros y no de inmigrantes, a pesar de que hasta el momento la mayor parte de los extranjeros establecidos en España, aunque no todos, son inmigrantes. Por ello en este estudio hablamos siempre de inmigrantes en lugar de hablar de extranjeros, como sería lo correcto si de otra perspectiva se tratara, ya que los resultados que aquí presentamos se refieren mayoritariamente al colectivo inmigrante.

Estructuramos la presente contribución comenzando por las fuentes disponibles para el estudio de la población inmigrante; seguimos con la evolución, origen y procedencia, en función de los continentes y de las naciones; para analizar después la distribución espacial de la población inmigrante, por barrios en la ciudad de Alcalá de Henares y en la de León, y su relación con el precio de la vivienda, para observar si existe una correlación positiva entre el volumen de inmigrantes y los precios del suelo, contribuyendo al debate sobre las interrelaciones que se puedan presentar. Lo que resulta de gran importancia para poder adecuar la gestión de los flujos migratorios a unas políticas migratorias, que tengan en cuenta tanto las necesidades de nuestro país como las del colectivo de inmigrantes que está en él.

\section{FUENTES DISPONIBLES PARA EL ESTUDIO DE LA DISTRIBUCIÓN ESPACIAL DE LA POBLACIÓN INMIGRANTE POR BARRIOS}

La abundancia y fiabilidad de los datos del Instituto Nacional de Estadística (INE), tanto en el Censo de Población del 2001 como en el padrón del año 2009, han aconsejado que se realice este estudio a partir de estas fuentes, ya que ofrecen un detalle a nivel de sección censal, que permite agrupar, no sin ciertas dificultades, los datos en los barrios establecidos oficialmente y vigentes en cada caso. Existen algunas dificultades conceptuales añadidas a estos hechos, como por ejemplo, la utilización de los datos sobre los extranjeros, que es la única fuente estadística demográfica posible, quitando las correcciones que se puedan realizar sobre los padrones, y por 
tanto, son la mejor aproximación a la inmigración. Todas ellas ya expuestas con detalle en estudios anteriores (Lázaro y González 2009:184). Esta población extranjera comprende un abanico muy amplio de situaciones sociales (jubilados y trabajadores, ricos y pobres, personas que residen legalmente en el país, ilegales, etc.) difíciles de abarcar exhaustivamente.

Otro hecho que dificulta este tipo de estudios, es que no todas las fuentes coinciden en los mismos datos para el colectivo de extranjeros, como se ha señalado en reiteradas ocasiones (Gonzalez y Lázaro, 2001 y 2007). Los censos de población ofrecen cifras superiores a las estadísticas del Ministerio de Trabajo e Inmigración (MTIN), ya que los primeros contabilizan los residentes, y no sólo la población legalmente establecida en España, como sucede con los datos del MTIN. Por otra parte, de todos es conocido que en nuestro país hay también una numerosa población inmigrante en situación ilegal, es decir, no registrada en las estadísticas oficiales. Su cuantía es desconocida, pero nada despreciable. Hay estudios sobre ella para algunas áreas concretas, como por ejemplo, el caso de Canarias del que se ocupan Domínguez, Díaz y Parreño, 2001.

En Alcalá de Henares en el año 2001, seis de cada 100 habitantes son extranjeros, hecho que no se produce en León hasta el año 2009, en el que en Alcalá de Henares son ya 20 de cada cien (tabla n ${ }^{\circ} 1$ ). El hecho de que Alcalá sea el municipio con más extranjeros de la Comunidad de Madrid, después del municipio de Madrid, supone que tiene una cifra superior de inmigrantes a la media nacional. Estudios recientes muestran el gran crecimiento de la inmigración en los años del boom inmigratorio en las ciudades del área metropolitana madrileña (Pozo y García Palomares, 2009)

Tabla 1. Incremento de la población (total y extranjera) en los municipios de Alcalá de Henares y León entre los años 2001 y 2009.

\begin{tabular}{|c|c|c|c|c|c|c|}
\hline \multirow{2}{*}{ Año } & \multicolumn{3}{|c|}{ Alcalá de Henares } & \multicolumn{3}{c|}{ León } \\
\cline { 2 - 7 } & $\begin{array}{l}\text { Población } \\
\text { total }\end{array}$ & $\begin{array}{l}\text { Población } \\
\text { extranjera }\end{array}$ & $\begin{array}{l}\text { Extranjeros } \\
\text { /total (\%) }\end{array}$ & $\begin{array}{l}\text { Población } \\
\text { total }\end{array}$ & $\begin{array}{l}\text { Población } \\
\text { extranjera }\end{array}$ & $\begin{array}{l}\text { Extranjeros } \\
\text { /total (\%) }\end{array}$ \\
\hline 2001 & 176.434 & 11.478 & 6,5 & 130.916 & 1722 & 1,32 \\
\hline 2009 & 204.574 & 42.263 & 20,66 & 134.305 & 8489 & 6,32 \\
\hline $\begin{array}{l}\text { Incremento } \\
\text { 2001 a 2009 }\end{array}$ & $15,95 \%$ & $268,21 \%$ & & 2,59 & 392,97 & \\
\hline
\end{tabular}

Fuente: Elaboración propia a partir de los datos del INE (Censo de 2001 y Padrón de 2009).

La ciudad de León no supera la media nacional en el número de inmigrantes ni en cifras absolutas ni en relativas. Lo que nos indica algo ya observado anteriormente, que la población inmigrante va a las grandes ciudades y a sus áreas de influencia (González y Lázaro, 2005:39). Tenemos así dos realidades claramente definidas, la de Alcalá de Henares, cuyo porcentaje de población extranjera se sitúa muy por en- 
cima de la media nacional (12,08 \%), cifrada en el padrón de 2009 en 20,66 \% y que podemos decir que tiene una tendencia alcista (los últimos datos de la Comunidad de Madrid la elevan en 2010 a 21,83 \%). Y la de León con un 6,32 \% que es claramente inferior a la media nacional, pero que coincide con la anterior en que también tiene una marcada tendencia alcista, en este caso producida o agudizada no sólo por el incremento de la población extranjera, sino por el decrecimiento de la población total, habiendo perdido más de 800 residentes en el año 2008. Esto explica un crecimiento lento e incluso negativo de la población, ya que la llegada de estos inmigrantes no contrarresta ni el envejecimiento que está padeciendo la ciudad de León, lo que ha dado lugar a un crecimiento negativo, ni la emigración desde la ciudad a otras zonas de España.

Otro hecho demográfico significativo de estos años, en general para España y para los municipios que nos ocupan, es el mayor ritmo de crecimiento de la población extranjera respecto al propio crecimiento de la población nacional. Esta realidad no se puede mantener para otras áreas, como por ejemplo el municipio de Madrid, que ha visto decrecer en el último año el número de extranjeros (Comunidad de Madrid, 2009). Tendrán que pasar algunos años para constatar si esta tendencia se extiende a Alcalá de Henares y a León, en donde de momento únicamente se constata la tendencia nacional a la ralentización del incremento del número de extranjeros, que en el último periodo interanual se cifra en un 103,95 \% para el caso de Alcalá de Henares y en un $102,52 \%$ para la ciudad de León. Mientras que para España en este mismo periodo 2008-2009 se cifra en un 105,87\%. Así, en ambos municipios ese crecimiento de la población extranjera en el último año ha sido inferior a la media nacional.

\section{EVOLUCIÓN, ORIGEN Y PROCEDENCIA DE LOS INMIGRANTES POR BARRIOS}

Se analiza el continente de procedencia y las nacionalidades predominantes en la distribución territorial de los inmigrantes en ambas ciudades. En todos los casos se sigue la tradicional tónica por la cual la mayoría de la inmigración procede de otros países de Europa, América del Sur y África (González y Lázaro, 2007:137) y su incremento desde el censo del 2001 ha sido el más acelerado, con diferencia, de toda la UE (Domínguez y Guerra, 2009:6), es decir, que las cifras de inmigrantes aunque son semejantes a las existentes en otros países de Europa de larga trayectoria de inmigración, como Alemania, Reino Unido o Francia, en España se han alcanzado en un tiempo record (Lázaro y González, 2009).

Se observa que las personas de cada nacionalidad tiendan a agruparse en un mismo vecindario, reflejándose mejor si trabajamos con unidades territoriales de menor tamaño que el barrio, como son las secciones censales. Esto ha sido ya constatado, entre otros autores, por González Yanci (2006).

Los extranjeros procedentes de Oceanía no ofrecen cifras significativas ni en la ciudad de Alcalá de Henares ni en la de León, por lo que no se han tenido en cuenta en el presente estudio. En ambos casos existe una menor proporción de población 
asiática, a pesar del reciente incremento de la población china y su tendencia a aumentar. Para el caso de Alcalá lo más característico será el predominio de la población europea rumana, que en la mayoría de los barrios suponen más de la mitad de la población inmigrante, mientras que en el caso de León será el predominio de la población procedente de América la que suponga más del 50\% de los extranjeros en la mayoría de los barrios.

\subsection{EL MUNICIPIO DE ALCALÁ DE HENARES}

En el caso del municipio de Alcalá de Henares, el continente de origen predominante en el citado municipio es el europeo, y dentro del mismo las nacionalidades que tienen mayor peso son los rumanos, polacos, búlgaros y ucranianos. En segundo lugar predominan los extranjeros procedentes del continente americano, destacando los colombianos y ecuatorianos. Y por último los africanos. En el año 2001 tenían un claro predominio los marroquíes, hoy desplazados por los nigerianos que se concentran en su mayor parte en el barrio de Reyes Católicos. A una gran distancia están los asiáticos, si bien es verdad que en 2009 el colectivo chino ha incrementado por ocho su contingente, aunque todavía no tiene el peso específico de otras nacionalidades, ocupando en importancia el undécimo lugar, según los datos del padrón de 2009.

Figura 1. Distribución del número de extranjeros por barrios en Alcalá de Henares, según el continente de procedencia en 2001 y 2009.

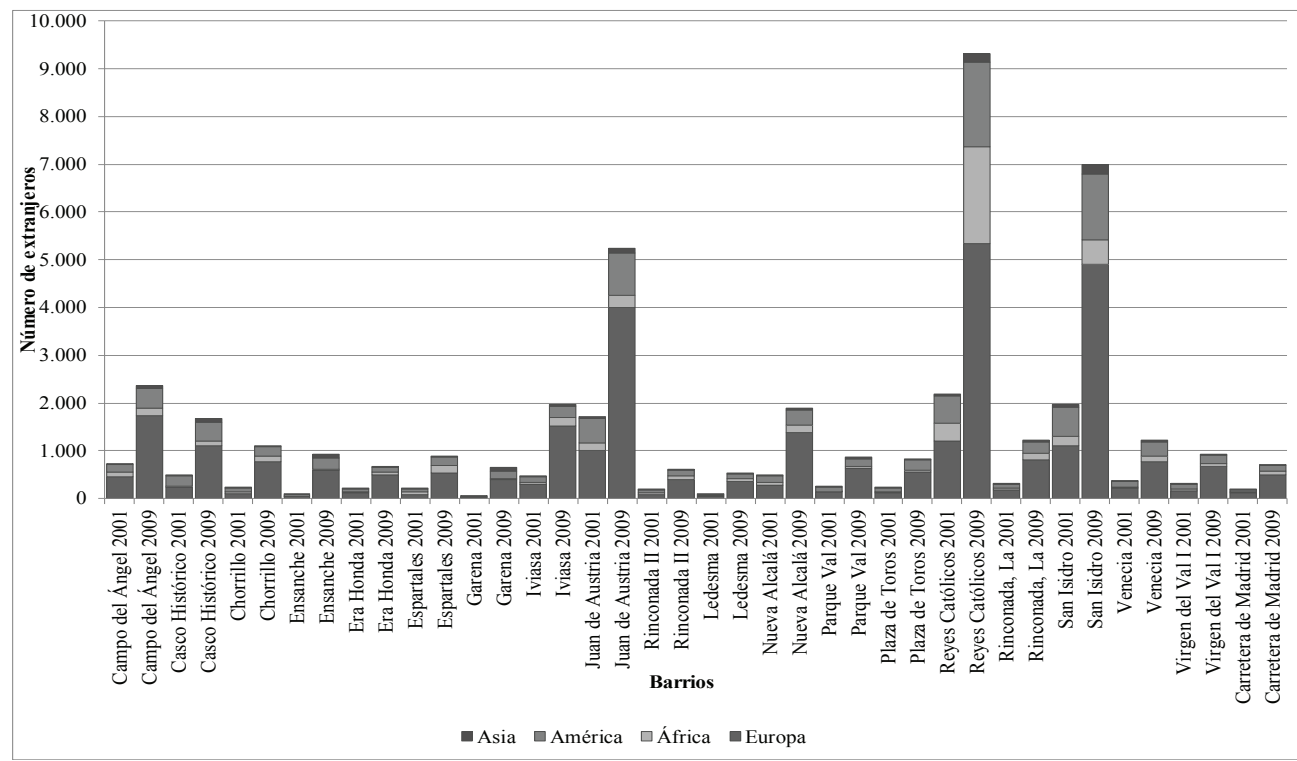

Fuente: Elaboración propia a partir de los datos del INE. 
En la figura $1^{2}$ se puede observar no sólo el número total de inmigrantes por barrios tanto en el año 2001 como en el 2009 (que se representa mediante las barras), sino el continente de procedencia de los mismos. Esto nos permite por un lado el crecimiento de la inmigración en esos años, hasta el año 2009, ya que en la actualidad se ha estancado el número de inmigrantes existentes en España. Si bien Alcalá de Henares sigue siendo un municipio en el que la afluencia inmigratoria ha sufrido una fuerte ralentización, pero en ningún caso ha desaparecido, no en vano se trata del segundo municipio de la Comunidad de Madrid en importancia por volumen de inmigrantes.

El predominio de la población europea en 2009 tiene un hecho muy destacable, el de que los rumanos constituyan más de la mitad de la población extranjera en el municipio (figura 2), mientras en el censo de 2001 sólo suponían la cuarta parte del mismo. Las grandes concentraciones de esta población se producen en los barrios en los que los extranjeros tienen un mayor peso, como son Reyes Católicos, San Isidro y Juan de Austria. Si bien el barrio de Reyes Católicos destaca también por su concentración de población africana y americana, lo que coloca a este barrio como el de mayor variedad racial de todo Alcalá, seguido, a una cierta distancia, por el barrio de San Isidro.

Figura 2. Distribución del número de extranjeros por barrios en Alcalá de Henares, según las nacionalidades principales de origen en el padrón de 2009.

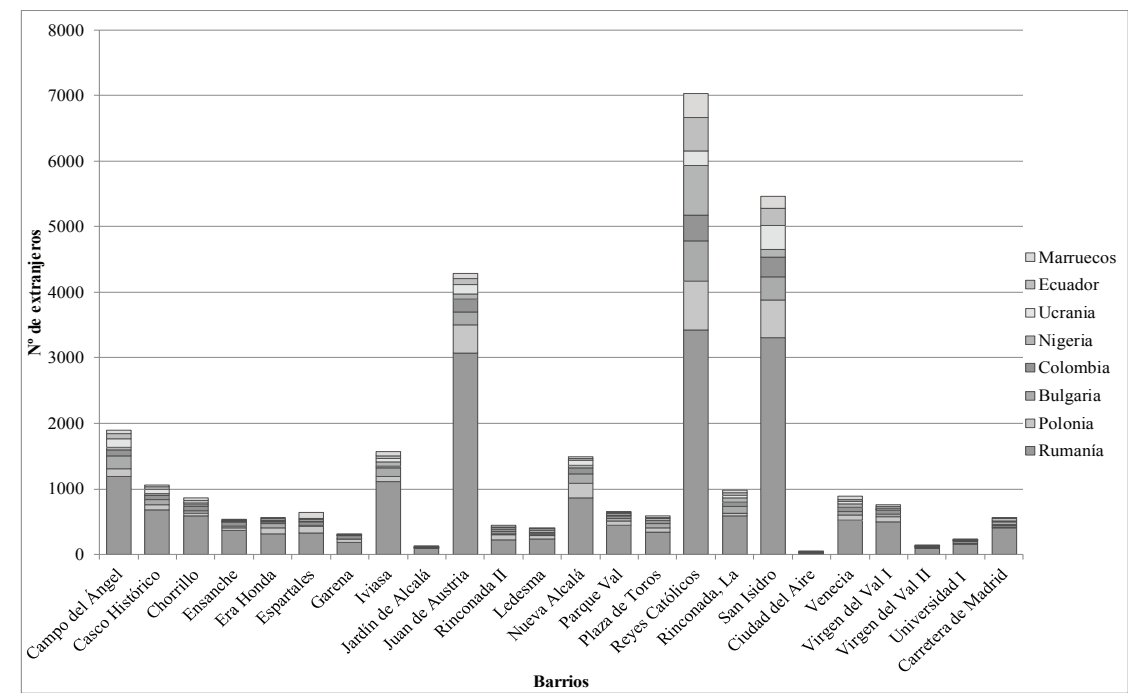

Fuente: Elaboración propia a partir de los datos del INE.

\footnotetext{
${ }^{2}$ Para facilitar la legibilidad del gráfico de la figura $\mathrm{n}^{\mathrm{o}} 1$ sólo se han representado los barrios que tienen más de 300 extranjeros en 2009. Para cada barrio se establecen dos columnas, una que representa todos los extranjeros en el año 2001 dividida en distintos colores en función del continente de procedencia y otra con la misma realidad en el año 2009, de esta forma se puede constatar claramente el volumen de crecimiento de los extranjeros en cada uno de los barrios de la ciudad entre uno y otro año.
} 
En la figura 2 se han representado las ocho nacionalidades mayoritarias en el municipio, es decir, aquellas que cuentan con más de 2000 extranjeros en el total del municipio. En su conjunto suponen más de las tres cuartas partes de la población extranjera. Si bien la ciudad cuenta con más de una treintena de nacionalidades distintas. La leyenda comienza con la más minoritaria de las ocho que es Marruecos y termina con la nacionalidad predominante, que es Rumanía, seguida de Polonia, Bulgaria, Colombia, Nigeria, Ucrania y Ecuador.

\subsection{EL MUNICIPIO DE LEÓN}

En la ciudad de León la tendencia en todos los barrios, como en el caso de Alcalá, es la de aumentar el peso de su población inmigrante (figura 3). Tanto en el 2001 como en el 2009 era Crucero-Pinilla el barrio que más inmigrantes tenía, seguido de La SalLa Vega. Chocantemente, a pesar de la centralidad y del nivel de vida del Ensanche, éste es uno de los barrios con mayor porcentaje de inmigrantes, tanto en el 2001 como en el 2009, situándose en ambos casos en tercer lugar, debido a que los edificios más antiguos y sin remodelar, se van ocupando por esta población en régimen de alquiler.

Figura 3. Distribución del número de extranjeros por barrios en León según el continente de procedencia en 2001 y 2009.

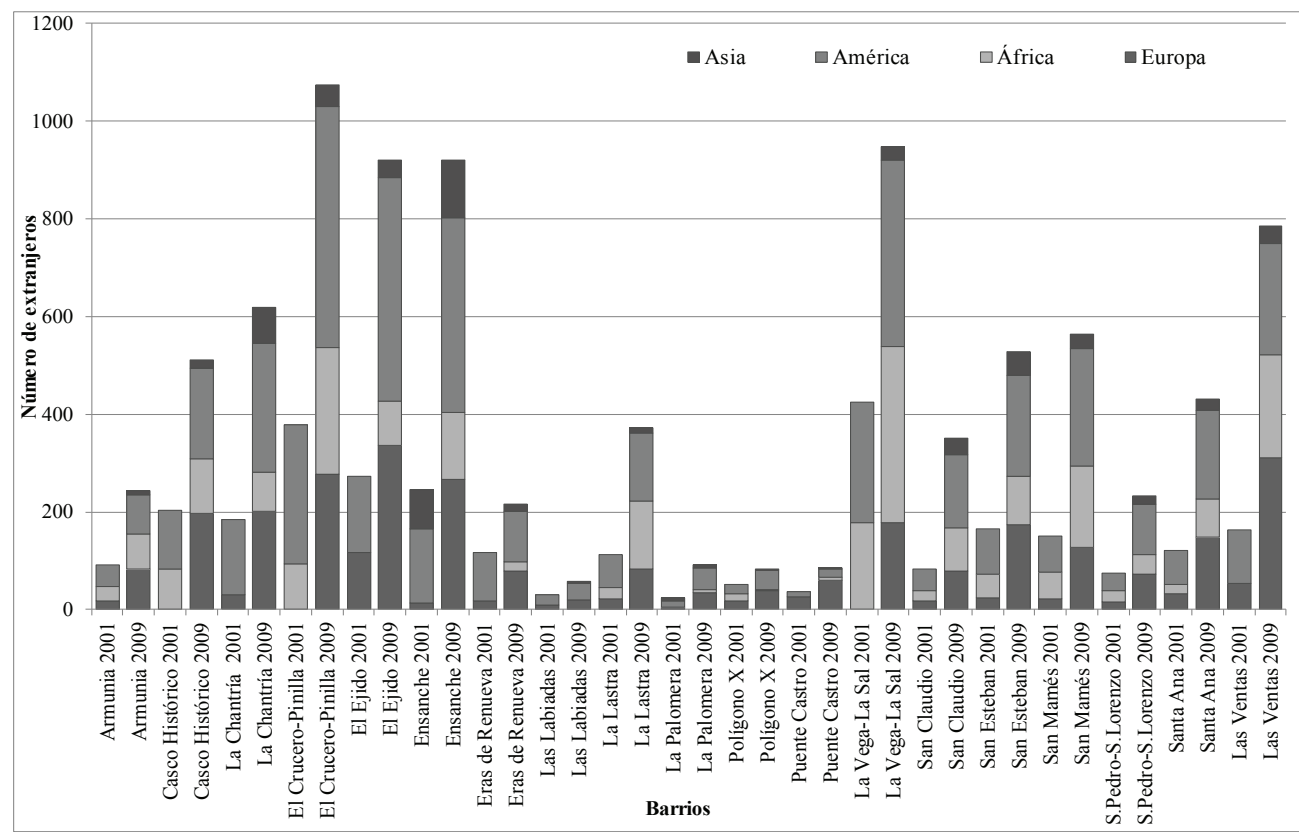

Fuente: Elaboración propia a partir de los datos del INE. 
El orden de importancia de la población extranjera por continentes es inverso al de Alcalá, predominando la población procedente de América y no de Europa, que se encuentra a cierta distancia de la anterior. La población europea se sitúa, principalmente, en los barrios más acomodados y de mayor nivel de vida, aunque los rumanos son mayoritarios en el barrio de Las Ventas. La nacionalidad predomínate dentro de los africanos es la de Marruecos en toda la ciudad, que se localizan en barrios como El Crucero-Pinilla, La Sal-La Vega o Las Ventas. La población asiática que en 2001 solo estaba presente en la Palomera, en el 2009 empieza a tener una creciente importancia y está presente en casi todos los barrios.

Como en el caso de Alcalá, y para que su representación gráfica sea más clara, se han tomado para León únicamente aquellas ocho nacionalidades que suponen las tres cuartas partes de la población extranjera según el padrón de 2009 (figura 4). La nacionalidad que predomina es la marroquí, seguida de la rumana, colombiana, dominicana, búlgara, brasileña, ecuatoriana y, por último, la nacionalidad más minoritaria de las ocho señaladas es la china. No obstante, los ecuatorianos han perdido importancia relativa desde el censo de 2001.

Figura 4. Distribución del número de extranjeros por barrios en León, según las nacionalidades principales de origen en el padrón de 2009.

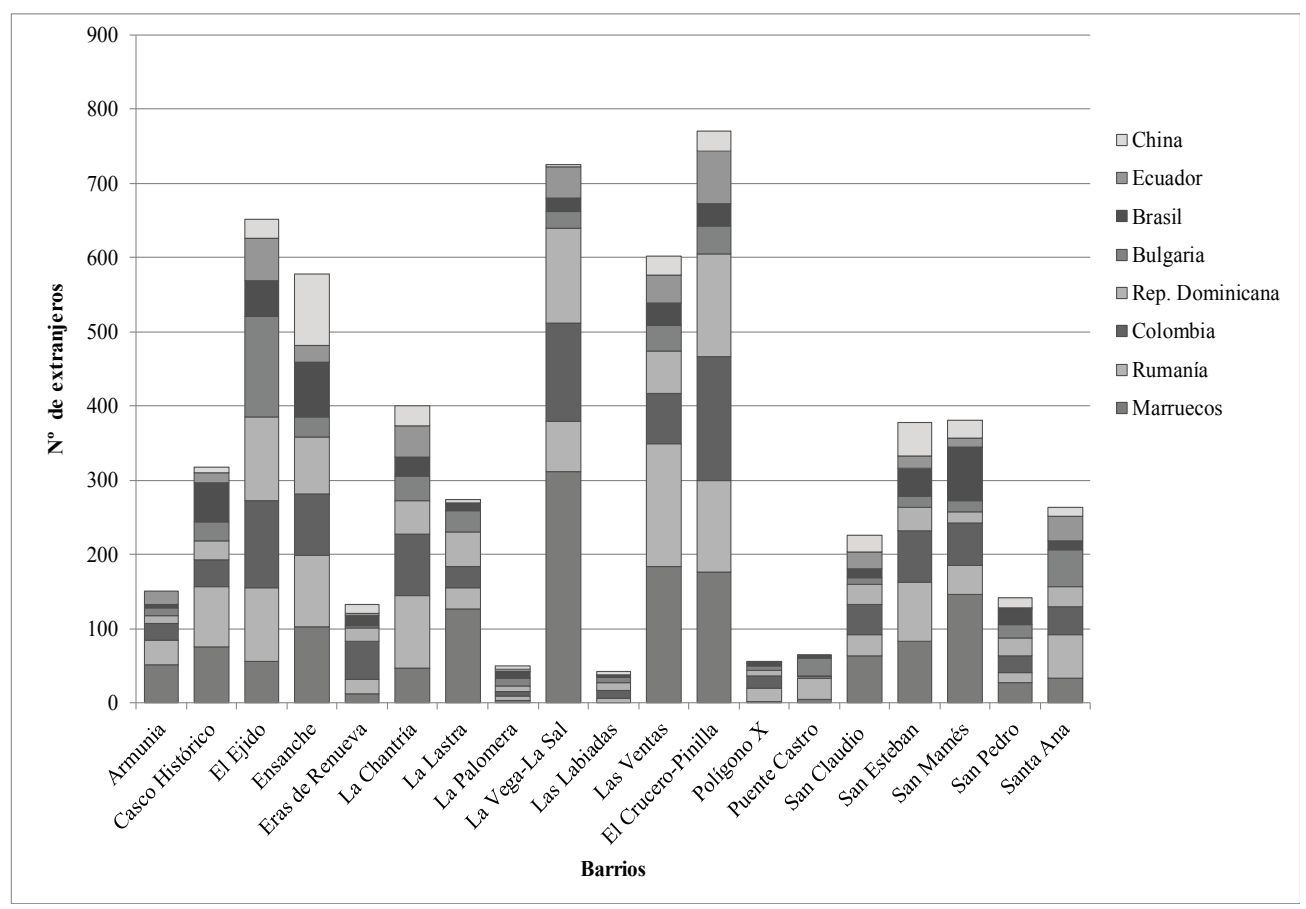

Fuente: Elaboración propia a partir de los datos del INE. 
En el cómputo global de la ciudad y con una presencia significativa, desde el censo de 2001, destacan los marroquíes, rumanos y colombianos. Los colombianos tienen un gran peso relativo, en la mayoría de los barrios, a excepción de Armunia, La Vega-La Sal, Polígono X y Casco Antiguo donde son superados por los marroquíes, que han aumentado notablemente, como por ejemplo en La Vega-La Sal que se han duplicado. En el 2009, los rumanos tienen una representación muy inferior a la de la ciudad de Alcalá de Henares, en dónde son predominantes. Los dominicanos han aumentado su representación en El Ejido, donde es casi la etnia dominante, después de la nacionalidad búlgara. La población china está a punto de convertirse en la etnia mayoritaria del Ensanche -donde los precios de las viviendas son más altos-, aunque actualmente debido al incremento en la ciudad de esta nacionalidad está presente en casi todos los barrios.

\section{LA DISTRIBUCIÓN ESPACIAL DE LA POBLACIÓN INMIGRANTE Y SU RELACIÓN CON EL PRECIO DE LA VIVIENDA POR BARRIOS EN ALCALÁ DE HENARES Y EN LEÓN}

Una vez observadas las diferencias en el porcentaje de población extranjera entre ambas ciudades y su origen en los distintos barrios vamos a cartografiar su distribución espacial y los precios de las viviendas en esos mismos vecindarios o barrios. No entramos en las dificultades para la obtención de los precios reales de las viviendas en España analizadas pormenorizadamente en estudios anteriores (Lázaro 1995, González y Lázaro, 2006) ni en el mercado de alquileres ampliamente trabajado en otros países en donde predomina el alquiler sobre la propiedad (Sanz, 2007), cuando en España tanto entre la población nacional como en la inmigrante la compra de la residencia permanente es un paso casi obligado (Pareja-Eastaway, 2009). Para ello hemos realizado un mapa para cada una de las ciudades en el que se cartografían el número de inmigrantes extranjeros en cifras absolutas por barrios, estableciendo el mismo valor de intervalo para poder realizar comparaciones entre ambas. Y también las cifras relativas correspondientes al porcentaje de la población extranjera respecto a la población total igualmente para cada barrio, que se representan mediante círculos proporcionales (figuras 5 y $7 \mathrm{a}$ ).

En ambos casos, podemos señalar como los barrios con un número más elevado de extranjeros son aquellos más cercanos al centro, céntricos o mejor comunicados o con unos precios de las viviendas más bajos, lo que otros autores han constatado ya para otras ciudades, como por ejemplo hace A. Zárate (2002) para la ciudad de Madrid.

Para comprobar estos hechos en las ciudades de Alcalá de Henares y de León, se ha realizado un mapa con los precios de las viviendas tomando más de 4000 anuncios inmobiliarios diferentes para el periodo 2001 a 2009, con la finalidad de alcanzar el mayor número posible de ellos y obtener así unas medias para cada barrio significativas. Se ha observado que aquellos barrios con una mayor oferta de viviendas son los que tienen un mayor número de extranjeros. Esto se puede explicar por el asenta- 
miento reciente de los mismos en los lugares en los que, por razones diversas que no sólo responden a las nuevas construcciones, hay disponibilidad de viviendas.

Se trata de analizar las diferencias en los precios de mercado de la vivienda plurifamiliar de segunda mano, que es la que aglutina una mayor oferta, entre los distintos barrios, por lo que se han cartografiado los precios en euros constantes del 2009 por metro cuadrado ${ }^{2}$.

Otro hecho a tener en cuenta es la dificultad de obtener precios reales o de mercado, ya que el acceso a ellos es prácticamente imposible. Por ello se ha optado por tomar los precios de la oferta de vivienda de segunda mano que se publican en la prensa de referencia para estos temas en esos años en cada localidad (el semanario "CAMBIHenares" para el caso de Alcalá de Henares y el periódico "Diario de León" para la ciudad de León) o bien en una de las Web de mayor relevancia (Idealista.com).

Podemos decir que los barrios que se consideran más caros (cascos históricos y ensanches de las ciudades) son los que tienen menos fluctuaciones del precio a lo largo del tiempo, al igual que sucede con aquellos que resultan más baratos (Armunia y La Sal/Vega, en León o Reyes Católicos, Nueva Alcalá o Iviasa en Alcalá de Henares) (figuras 6 y 7b). Por lo que podemos afirmar que las diferencias de precios entre los barrios tienden a mantenerse. Si bien, en este caso, no se trata de un estudio de la evolución de esos precios en función de los barrios, cuyos múltiples matices exigirían un trabajo aparte, sino de un estudio sobre las diferencias existentes entre los distintos barrios y su relación con la localización espacial de la población inmigrante.

\subsection{LA CIUDAD DE ALCALÁ DE HENARES}

En el caso de Alcalá de Henares los barrios con más de la cuarta parte de población extranjera sobre su población total son por orden (figura 5): Juncal, San Isidro, Carretera de Madrid, Era Honda, Juan de Austria, Reyes Católicos y Plaza de Toros.

Entre ellos están incluidos los tres barrios (Reyes Católicos, San Isidro y Juan de Austria) que desde el censo del 2001 reúnen a más de la mitad de la población inmigrante que reside en Alcalá. Todos estos barrios están próximos al centro y muy bien comunicados tanto por el ferrocarril, como es el caso del barrio de San Isidro, como por los autobuses interurbanos que tienen algunas de sus paradas principales en los barrios de Reyes Católicos y Juan de Austria.

En relación con los precios de la vivienda en los mismos cabe señalar que ninguno de estos barrios están entre los que tienen los precios medios más altos de la ciudad de Alcalá de Henares (figura 6). El barrio de Reyes Católicos, con más de 9.000 extranjeros, es el que se queda más alejado del centro de todos ellos y con unos precios medios de la vivienda inferiores a los otros tres barrios y a la media de la ciudad. La oferta inmobiliaria existente en este barrio dobla la de cualquier otro del municipio. Además la importancia del fenómeno inmigratorio en el mismo se refuerza por la gran densidad de inmigrantes en los barrios inmediatamente contiguos (Carretera de Madrid, Juncal, Era Honda, La Rinconada, y Nueva Alcalá), que en todos los casos superan el $20 \%$ de extranjeros sobre la población total. Así, para el caso de 
este barrio, se cumplen las conclusiones realizadas en estudios realizados para otras ciudades, como Dublín (Fahey y Fanning, 2010) o el Condado de Santa Clara en California (Cervero y Duncan, 2004), por los cuales la diversidad racial y la segregación socio-espacial de un barrio es un indicador que se correlaciona con los precios más bajos de las viviendas.

Figura 5. Distribución de la población extranjera en Alcalá de Henares.

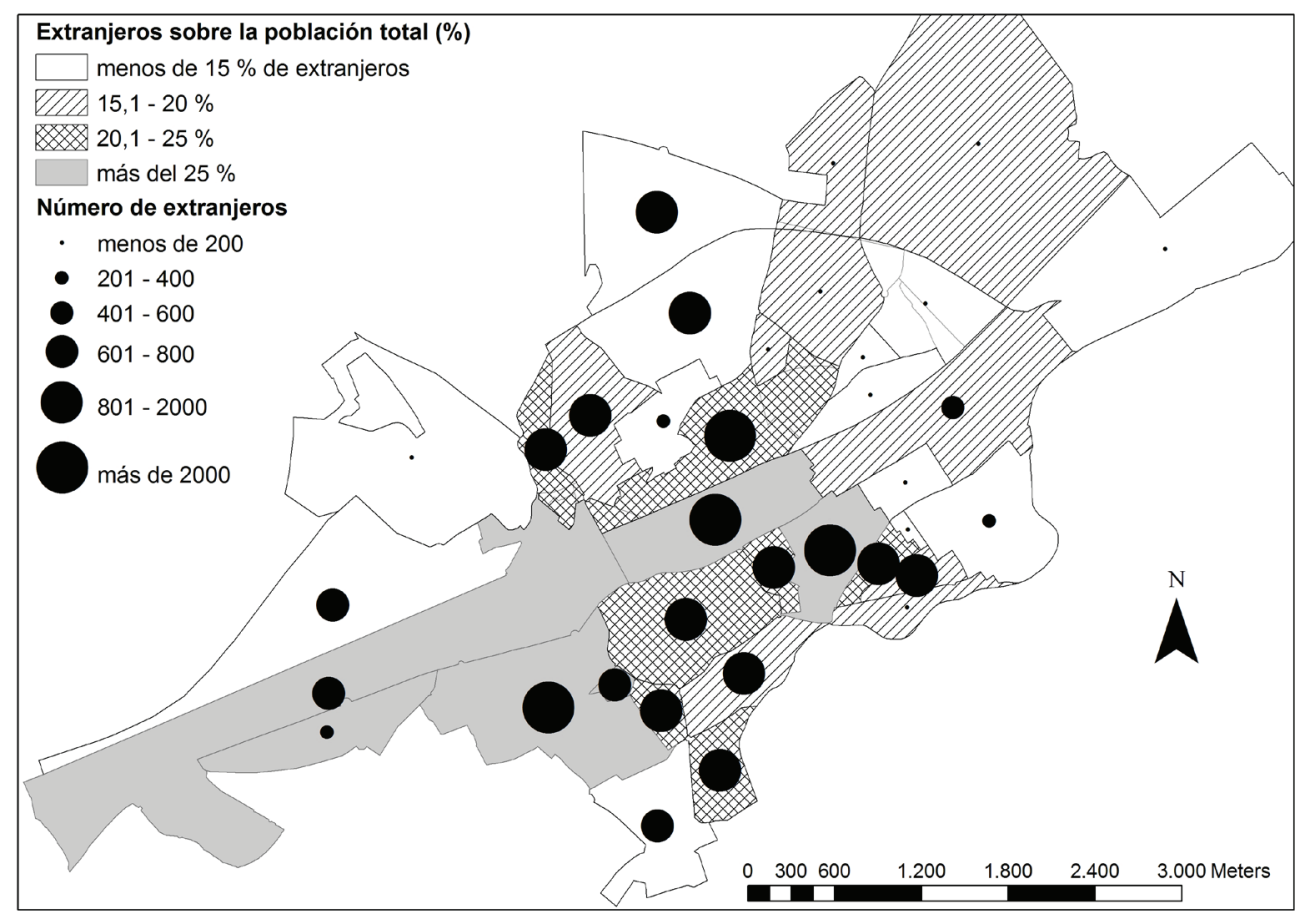

Fuente: Elaboración propia realizada con ArcGIS a partir del Padrón de habitantes, 2009 (INE).

Le sigue el barrio de San Isidro, con unos 7.000 extranjeros. Este barrio es uno de los que tiene una mayor variedad en los precios de la vivienda, ya que mientras el paseo de la Estación es una de las zonas más caras de la ciudad (González y Lázaro, 2009) las calles interiores del barrio cuentan con viviendas de distinta calidad algunas de ellas muy degradadas y con algunos bloques de pisos sin ascensor. Todo esto explica un precio de la vivienda por encima de la media de la ciudad y una gran diversidad socioeconómica en la población residente en el barrio.

El barrio de Juan de Austria cuenta también con una gran variedad de viviendas construidas en distintas épocas y un precio medio que está ligeramente por encima 
de la media de la ciudad. Su volumen de población extranjera es inferior a los dos barrios anteriores superando los 5.000 residentes extranjeros.

Figura 6. Distribución de los precios de la vivienda plurifamiliar de segunda mano por barrios en Alcalá de Henares (en precios constantes de 2009) ${ }^{3}$.

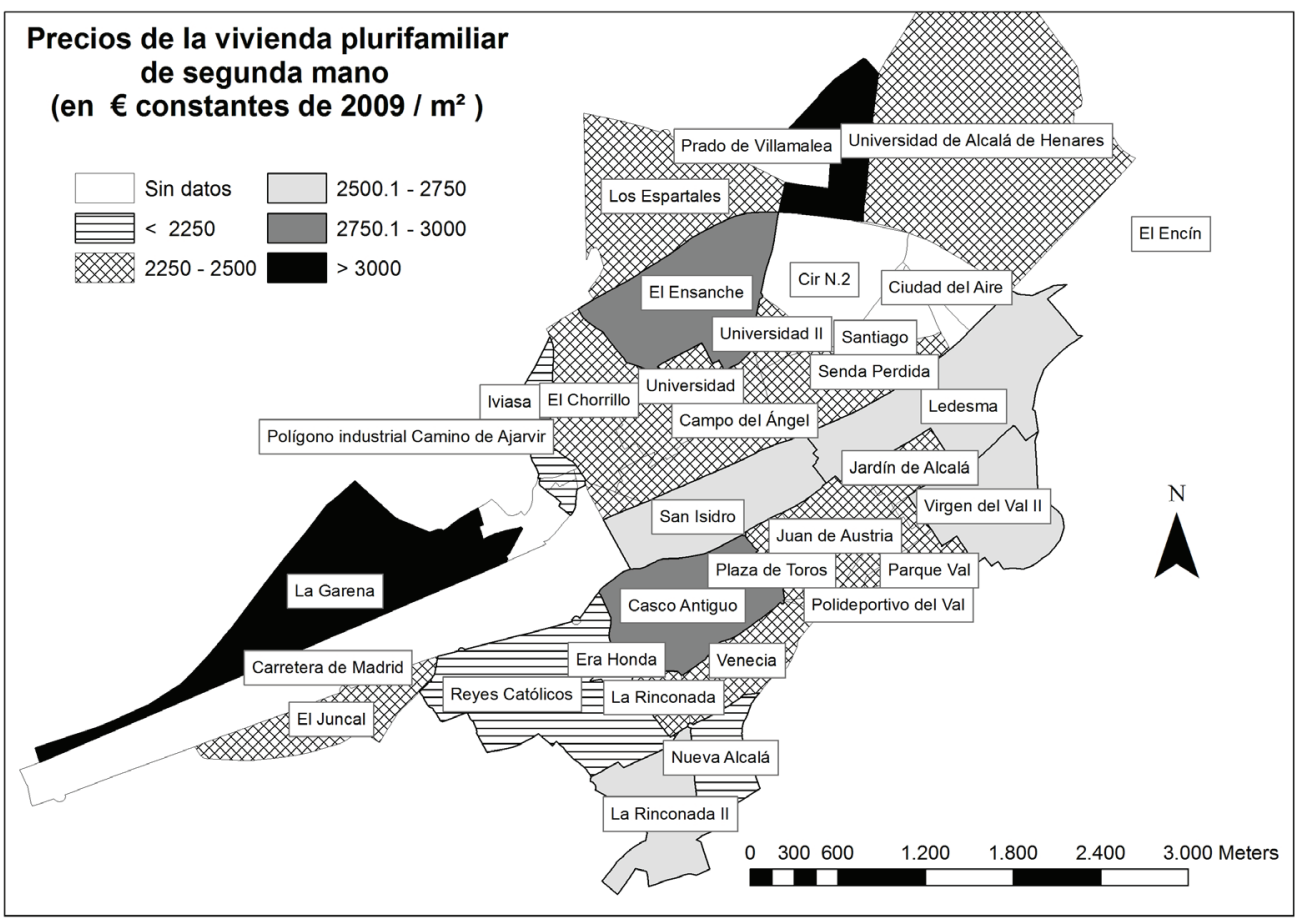

Fuente: Elaboración propia realizada con ArcGIS a partir de los anuncios inmobiliarios del semanario CAMBIHenares y de la página web idealista.com.

El Campo del Ángel es el barrio que le sigue con un número elevado de inmigrantes (más de 3.000) y una gran variedad en su parque de viviendas, lo que hace que los precios medios de la misma estén ligeramente por debajo de la media para la ciudad.

Los barrios de reciente edificación suponen una realidad opuesta a la observada en los barrios analizados. Se trata de los barrios con los precios de las viviendas más elevados y que cuentan con un menor porcentaje de inmigrantes entre su población,

${ }^{3}$ Los precios constantes del 2009 han sido obtenidos mediante la media aritmética para cada barrio de los precios de los años 2001, 2004, 2007, 2008 y 2009 deflactados con el índice de precios al consumo correspondiente a cada año. 
ninguno de ellos llega a tener mil extranjeros residiendo en él. Estos son La Garena, Ensanche, La Rinconada II, Espartales, Polígono industrial de Ajalvir, etc.

El barrio Ledesma tiene un creciente número de viviendas nuevas que se explican por distintas iniciativas inmobiliarias, como Pryconsa, sobre un barrio de tradicional uso industrial. Este barrio cuenta con un mayor número de extranjeros en cifras absolutas y relativas que otros barrios con precios semejantes. Esto se explica porque en este barrio existen algunas áreas industriales y de vivienda degradadas.

Otros barrios con un bajo porcentaje de extranjeros en números absolutos y relativos se debe a su vinculación con las actividades militares, que generan barrios con las viviendas de las personas vinculadas a las mismas (Ciudad del Aire, Santiago y Senda Perdida) y algunos barrios considerados, tradicionalmente, en Alcalá como de gran calidad en los que frecuentemente existe una tradicional vivienda unifamiliar, como por ejemplo, Virgen del Val II.

El elevado número de inmigrantes en el casco antiguo y en otros barrios céntricos, aunque en ningún caso se aproximen en cifras al de Reyes Católicos, se explica por la segmentación del mercado inmobiliario, del que se deriva en parte, la dificultad de establecer precios medios para la vivienda (Lázaro, 1990, 1995). Así, en esos barrios alternan las viviendas de calidad con otras deterioradas o de baja calidad, que son las efectivamente ocupadas por los inmigrantes. La ocupación de los edificios de peor calidad dentro de los barrios permite pagar menores precios en la vivienda, lo que se puede derivar en un mayor ahorro que incremente la cuantía del envío de las remesas, principal objetivo del inmigrante.

La ausencia de datos en los precios de algunos barrios, como Carretera de Madrid, responde principalmente a que se trata de un área de tradicional uso industrial que está iniciando una transformación a uso residencial todavía no consolidado, o de un área de vivienda protegida, y que por tanto no puede ser vendida en el libre mercado.

\subsection{LA CIUDAD DE LEÓN}

En el caso de la ciudad de León, la población inmigrante se establece en las áreas más céntricas (figura 7a), en torno al Casco Histórico, pero sin ser éste barrio el que cuenta con una mayor ocupación.

Se observa, como los inmigrantes, en muchos casos, sustituyen en las viviendas más antiguas de los barrios céntricos a las clases medias, que se desplazan a residir en la periferia, en polígonos de nueva construcción. En ninguno de los barrios tenemos una ocupación que supere los 2.000 inmigrantes, como sucede en el caso de Alcalá de Henares, lo que indica que la importancia relativa de la inmigración en la ciudad de León es muy inferior a la de la ciudad de Alcalá de Henares.

El mayor volumen de inmigrantes en la ciudad de León, en el año 2001, se concentra, como en el caso de Alcalá, en los barrios que cuentan con unos precios de la vivienda inferiores a la media de la ciudad (figura $7 \mathrm{~b}$ ), y cercanos al centro, que son principalmente La Vega- La Sal seguido de cerca por el barrio de Pinilla- El Crucero. La Lastra, a pesar de ser un barrio con un gran porcentaje de extranjeros, no tiene una 
gran aportación en cifras absolutas, ya que hay muy poca población nacional, debido a que es un polígono que se estaba desarrollando actualmente y con la crisis económica que ha afectado a la construcción se ha paralizado.

Figura 7. Población extranjera y precios del suelo en el municipio de León.

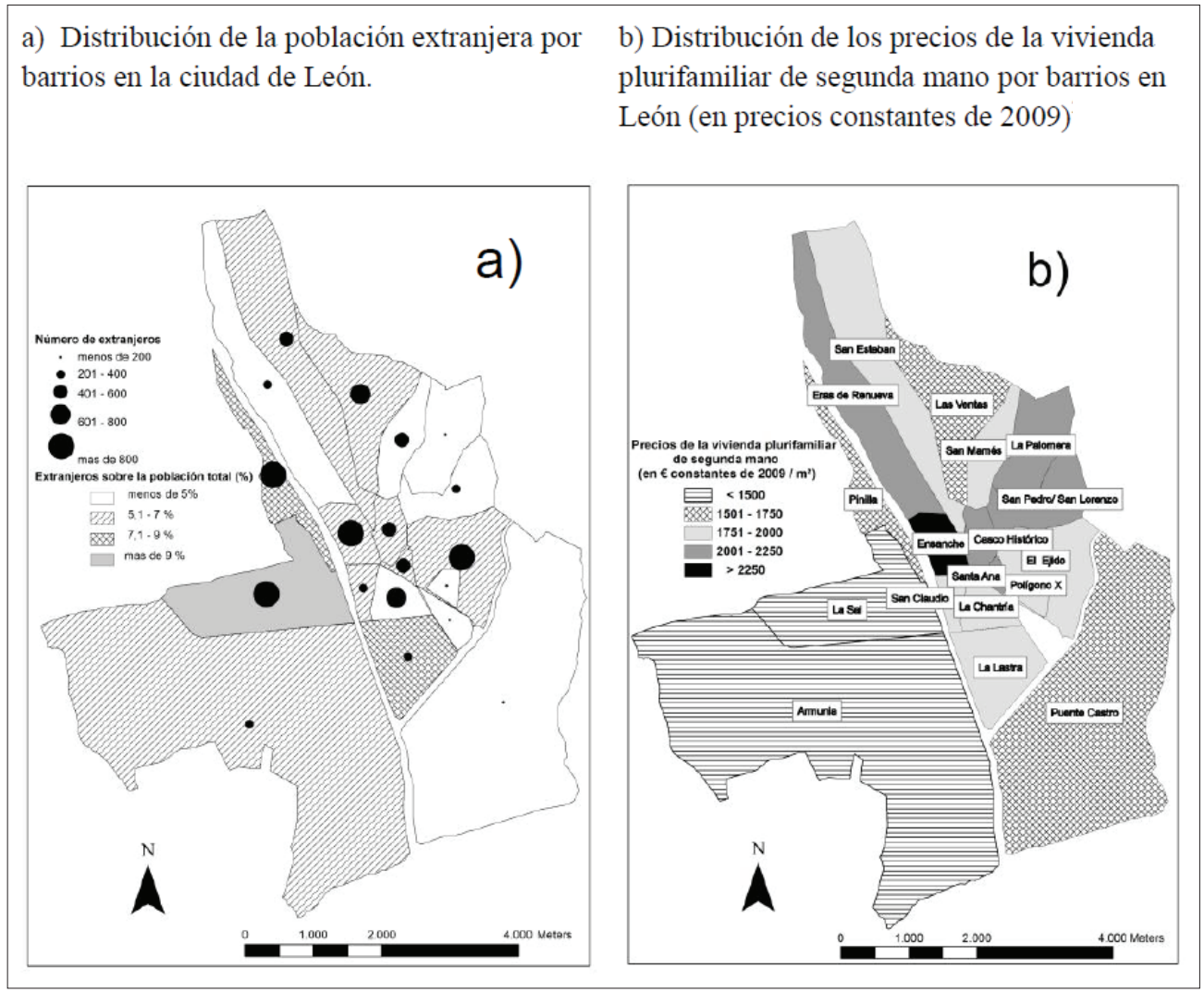

Fuente: Elaboración propia realizada con ArcGIS a partir de los datos del Padrón de habitantes, 2009 (INE) y los anuncios inmobiliarios del "Diario de León" y de la página Web de Idealista.com.

En menor medida los extranjeros también se concentran en los barrios de El Ejido y Ensanche, que ya cuentan con unos precios superiores a la media de la ciudad. Esto se puede explicar no solo por la nacionalidad de los extranjeros, algunos de ellos empresarios, y por la existencia de viviendas degradadas en ambos barrios, sino también por la segmentación del precio del mercado inmobiliario. Así, en estos barrios alternan las viviendas de calidad con otras deterioradas o de baja calidad, que son 
ocupadas por los inmigrantes. Este hecho es extrapolable también al barrio Eras de Renueva, que es otro de los barrios con precios de viviendas elevados. Sin embargo, hay que señalar que una gran parte de la población china, que reside en este barrio y en el Ensanche, tiene un nivel de renta superior al del resto de los inmigrantes, ya que regentan sus propios locales como autónomos de la restauración o de los bazares.

En 2009, la población de inmigrantes se ha triplicado con respecto al 2001, y en algunos barrios ha supuesto un crecimiento espectacular. Los que más han incrementado el número de extranjeros son: El Crucero-Pinilla, La Sal-La Vega, El Ensanche, Las Ventas y San Mames. Todos ellos cuentan con viviendas degradadas, algunos bloques de pisos sin ascensor y una gran variedad de viviendas construidas en distintas épocas, lo que explica una gran diversidad socioeconómica de la población residente en estos barrios. Los polígonos de más reciente edificación como $\mathrm{La}$ Palomera, el polígono de San Pedro o el Polígono X (barrio residencial de nueva construcción en los años ochenta) es en los que menos ha aumentado y no superan los 500 extranjeros.

Los datos recogidos en la figura 7 corroboran que los dos barrios con los precios más altos de las viviendas son el Ensanche y el Casco Histórico, como se ha constatado en la práctica totalidad de los estudios sobre el precio del suelo (González y Lázaro, 2006, 2009 a). En el barrio del Ensanche el número de inmigrantes es superior a la media de la ciudad, mientras que en el casco histórico es inferior, hecho que indudablemente contrasta con lo detectado para otras ciudades españolas, como por ejemplo, Alcalá de Henares que el Casco Histórico tiene un porcentaje ligeramente superior a la media de la ciudad. Esto se puede explicar por el estado de las viviendas existentes en cada uno de los barrios. Así, en el Casco Histórico de León se han rehabilitado muchas viviendas o se han construido de nuevo, pero con alturas de dos o tres plantas, por tanto con una densidad menor a la del Ensanche, que han sido ocupadas por matrimonios jóvenes o personas que viven solas nacionales, de ahí el menor peso de los extranjeros que se ubican en las viviendas más degradadas.

\section{LA CORRELACIÓN ENTRE EL VOLUMEN DE INMIGRANTES Y LOS PRECIOS DEL SUELO POR BARRIOS}

Algunos autores, como Sosvilla (2008) afirman que el incremento de la demanda de vivienda, como consecuencia de la inmigración, ha supuesto una subida en el precio de las viviendas en las ciudades con mayor afluencia de inmigrantes. No podemos negar que la inmigración, como una parte de la demanda existente haya podido influir, si consideramos el municipio en su totalidad, pero no podemos ratificar que esto haya podido suceder en todos los barrios, ni que haya sido un factor decisivo de las fluctuaciones en los precios de los mismos.

Para profundizar en este hecho se han correlacionado para ambas ciudades (Alcalá y León), siguiendo el coeficiente de correlación de Spearmn, la variación de ambas variables (población y precios de la vivienda) entre el año 2001 y el 2009 (7 DED(D) 2). En el caso de los municipios tomados globalmente, no obtenemos tanta riqueza 
de matices - la media de Alcalá por barrios sale 0,27 y la de León 0,26 - como si lo aplicamos a los distintos intervalos de precios que hemos establecido para cada una de las ciudades, siendo la correlación media de 0,6 para ambas ciudades. Su análisis por barrios se ha aplicado a los distintos intervalos de precios.

En ambos casos se ha descartado el barrio con precios más altos, en el caso de Alcalá de Henares, el barrio de La Garena, por ser un barrio de reciente construcción que se crea como segundo centro de la ciudad, con un elevado número de oficinas y grandes almacenes y Prado de Villamalea, que no cuenta con datos suficientes como para poder tener resultados significativos en este estudio, y además, también es un barrio de reciente creación. En el caso de León se ha desechado el barrio del Ensanche, que es el de los precios más altos y está él sólo en un intervalo, lo que reduciría su significación.

Tabla 2. Correlación de Spearman tomando el incremento de 2001 a 2009 de las dos variables señaladas, el precio de la vivienda y el número de inmigrantes según los intervalos de precios establecidos previamente en la cartografía y los barrios.

\begin{tabular}{|l|c|l|}
\hline Municipio & Intervalos de precios por barrios & $\begin{array}{c}\text { Correlación } \\
\text { Spearman }\end{array}$ \\
\hline \multirow{2}{*}{ León } & $2001-2250 € / \mathrm{m}^{2}$ & 0,142857143 \\
& $1751-2000 € / \mathrm{m}^{2}$ & 0,371428571 \\
& $1500-1750 € / \mathrm{m}^{2}$ & 0,5 \\
& $<1500$ & -1 \\
\hline \multirow{2}{*}{ Alcalá de Henares } & $2751-3000 € / \mathrm{m}^{2}$ & 1 \\
(Madrid) & $2501-2750 € / \mathrm{m}^{2}$ & -1 \\
& $2250-2500 € / \mathrm{m}^{2}$ & 0,160839161 \\
& $<2250$ & $-0,5$ \\
\hline
\end{tabular}

Fuente: Elaboración propia a partir de los datos del INE y los anuncios inmobiliarios de la prensa.

Los resultados obtenidos son, aplicando el coeficiente de correlación de Spearman por tramos de precios medios, los más significativos de las opciones posibles. Con ello se desprende que los precios y las migraciones, para la ciudad de León, únicamente tienen una ligera correlación inversa más significativa en los barrios de menor precio de la vivienda. Por tanto, se puede corroborar que los precios de la vivienda 
han bajado en función del incremento del número de inmigrantes en estos barrios, desde el año 2001. Este hecho se ha constatado para otras ciudades, como Dublín o algunos barrios de California ((Fahey y Fanning, 2010; Cervero y Duncan, 2004). Esto se debe a la movilidad intraurbana, ya que los antiguos residentes, fruto de la bonanza económica de años atrás, se han ido a áreas residenciales de mejor calidad en las nuevas urbanizaciones de la periferia de la ciudad.

Los barrios con los precios de la vivienda más elevados, el Casco Histórico y el Ensanche (tanto para Alcalá como para León respectivamente), no han abaratado su precio porque se haya incrementado el número de inmigrantes en ellos -ya que ocupan las viviendas más degradas en alquiler-. Sin embargo, los barrios con precios de la vivienda por encima de la media de la ciudad sí se han visto influenciados por la inmigración, por lo que el incremento de los precios es inversamente proporcional al incremento de los extranjeros. Esto es lo que sucede en los barrios de San Isidro, Ledesma, Virgen del Val II y La Rinconada II, de Alcalá, lo que encierra realidades muy distintas, en el primer caso es un barrio céntrico con un número muy elevado de inmigrantes, Virgen del Val II es un barrio que tiene menos porcentaje de inmigrantes, y el último caso es un barrio que en su mayor parte se ha construido en los años noventa. En León coincide con La Vega-La Sal, El Crucero-Pinilla, Las Ventas, tradicionalmente barrios donde se han asentado los inmigrantes, y con el barrio de San Esteban más céntrico pero con un menor número de inmigrantes.

En el intervalo siguiente de precios, que es el que aglutina a la mayor parte de la ciudad no hay una correlación importante entre los precios de la vivienda y la inmigración. Este hecho coincide en León con los barrios de más reciente creación La Palomera, Eras de Renueva, San Pedro-San Lorenzo. También ocurre en algunos barrios con un gran peso de extranjeros, como puede ser el del Campo del Ángel en Alcalá y El Ejido y La Lastra en León, debido a la diversidad socioeconómica de estos barrios. Y por último, los barrios con los precios de la vivienda más baratos tienen una correlación moderada entre los precios de la vivienda y los extranjeros residentes en el barrio; son todos ellos barrios con un porcentaje de inmigrantes igual o superior a la media del municipio.

No vamos a entrar en los beneficios, en general, que ha supuesto la inmigración en España, ya que sobre ello se ha reflexionado en estudios anteriores y varios autores se han centrado en ello (Pereda, Actis y Prada, 2008; Lázaro y González, 2009). No obstante, tenemos que señalar que la inmigración ha constituido una inestimable demanda para las viviendas de menor calidad, de forma que este segmento inmobiliario, en régimen de alquiler, se ha visto beneficiado. Por tanto, el hecho de que sólo cerca del 18\% de los inmigrantes piensen en comprar una vivienda (Pereda, Actis y Prada, 2005) no ayuda al incremento de la demanda directa, pero si indirectamente, ya que puede haber inversores dispuestos a realizar esa compra para luego alquilar las viviendas. 


\section{CONCLUSIONES}

El fenómeno inmigratorio en la ciudad de León y su entorno, y en toda la Comunidad de Castilla y León en general, dista mucho de tener la intensidad que en otras comunidades españolas, como sucede con Madrid y su área de influencia, cuyo atractivo socioeconómico hacen de ella, la Comunidad Autónoma de mayor importancia inmigratoria de España, y Alcalá de Henares tiene un papel protagonista en ella.

En ambos casos, la población inmigrante que se establece en las áreas más céntricas lo hace en viviendas de precio asequible, que suelen ser las más deterioradas, como en la mayoría de los países, y además en áreas en las que los precios de la vivienda son más moderados. Así, en el caso de Alcalá hay un gran volumen de inmigrantes en el barrio de Reyes Católicos y sus alrededores, y en el caso de León, y por las mismas razones son los barrios de Pinilla, la Sal y sus alrededores los que cuentan con más población extranjera. Los extranjeros dedicados a actividades empresariales se establecen en las áreas más céntricas, algunos de ellos acceden a viviendas de precios elevados. Son los barrios con una elevada variedad de viviendas disponibles los que acogen a un mayor número de inmigrantes, ya que la mayor oferta inmobiliaria tiene una relación directa con una cierta importancia de la inmigración en la ciudad.

Hemos constatado como los precios medios de los barrios son un elemento importante para el establecimiento de la población inmigrante en los mismos, junto con las buenas comunicaciones urbanas e interurbanas, si bien su estudio no está exento de dificultades que se explican por un lado, por la segmentación del mercado inmobiliario y la dificultad de manejar precios reales de mercado y por otro, por la dificultad de contabilizar a los extranjeros ilegalmente asentados en España.

Actualmente, parece que asistimos a una ralentización del ritmo en el incremento de los inmigrantes en ambos municipios, siguiendo la tónica más generalizada de España. Aunque, no parece que se hayan frenado los flujos inmigratorios ni que hayan regresado masivamente a sus países de origen, quizás debido a que la crisis económica actual afecta al mundo entero, incluidos los países de origen de los inmigrantes, no obstante, algunas áreas del mundo están empezando a salir de ella antes que otras. También podemos señalar que el sector de la vivienda y sus precios están estancados.

\section{BIBLIOGRAFÍA}

AKBARI, A. y AYDEDE, Y. (2009): Effects of Immigration on Housing Prices in Canada, Atlantic Metropolis Centre, Working Paper No. 24-2009.

BODEGA, M. I., CEBRIÁN, J. A. y MARTÍN LOU, M. A. (2006): El crecimiento de la población extranjera en Madrid. Un episodio característico de la inmigración internacional en la España del cambio de siglo. Estudios Geográficos, LXVII, 261, p. 385-416. 
CEBRIÁN, J. A., BODEGA, M. I. Y MARTÍN LOU, M. A. (2008): La inversión migratoria en Europa. ARBOR Ciencia, Pensamiento y Cultura, CLXXXIV, 734 noviembre-diciembre, p. 1129-1146.

CERVERO, R. y DUNCAN, M. (2004): Neighbourhood Composition and Residential Land Prices: Does Exclusion Raise or Lower Values? Urban Studies, vol. 41, 299315.

COLEMAN, A. y LANDON-LANE, J. (2007): Housing Markets and Migration in New Zealand, 1962-2006, Reserve Bank Discussion Paper, DP2007/12.

COMUNIDAD DE MADRID. (2009): Informe demográfico de la población extranjera en Madrid. Junio 2009. Madrid: Observatorio de Inmigración- Centro de Estudios y Datos de la Consejería de inmigración y Cooperación.

COMUNIDAD DE MADRID. (2010): Informe de la Población Extranjera Empadronada en Madrid. Enero de 2010. Madrid: Observatorio de InmigraciónCentro de Estudios y Datos de la Consejería de inmigración y Cooperación.

DEGEN, K. y FISCHER, A. (2010) Immigration and Swiss House Prices. Swiss National Bank working papers 2010-16.

DOMÍNGUEZ MUJICA, J., DÍAZ HERNÁNDEZ, R. y PARREÑO CASTELLANO, J. M. (2001): Inmigración extranjera e integración. La inmigración irregular en Canarias (1999-2000). Las Palmas de Gran Canaria: Gobierno de Canarias.

DOMÍNGUEZ MUJICA, J y GUERRA TALAVERA, R. (2009): The Demographic Transition and Immigration Flows in Spain: a close relationship. Geographical Review of Japan Series B, 81(1), p. 1-15. The Association of Japanese Geographers.

FAHEY, T. y FANNING, B. (2010): Immigration and Socio-spatial Segregation in Dublin, 1996-2006. Urban Studies, vol 47 (8), p. 1625-1642.

GONZÁLEZ GONZÁLEZ, M.J. y LÁZARO Y TORRES, M. L. de. (2005): La localización de la población inmigrante en España a través del censo de la población de 2001. Principales características. Cuadernos geográficos de la Universidad de Granada, 36, p. 35-49.

GONZÁLEZ GONZÁLEZ, M.J. y LÁZARO Y TORRES, M.L. (2006): The trends towards the increase in housing prices in Spain. The scope of reinterpreting urban areas. In IGU, Urban changes in different scales: systems and structures. Universidad de Santiago de Compostela/IGU Commission on monitoring cities of tomorrow. Santiago, p.149-161.

GONZÁLEZ GONZÁLEZ, M.J. y LÁZARO Y TORRES, M. L. de. (2007): A review on the situation of immigration in Spain in the european context. International Review on Public and Non Profit Marketing, vol 4, $\mathrm{n}^{\mathrm{o}} 1 / 2$ (December 2007), $\mathrm{p}$. 133-147.

GONZÁLEZ GONZÁLEZ, M.J. y LÁZARO Y TORRES, M. L. de. (2010): Recent problems of the historic centres in Spain. Journal of US-China Public Administration, vol. 7 (6), Serial No.56, p. 82-92. 
GONZÁLEZ YANCI, M. P. (2006): De la España que emigra a la España que acoge. Un proyecto de reflexión sobre la emigración e inmigración en España a través de una exposición y un libro catálogo de la fundación Francisco Largo Caballero, Estudios Geográficos, LXVII, 261,p. 689-692.

GONZALEZ, L. y ORTEGA, F. (2009): Immigration and Housing Booms: Evidence from Spain. IZA Discussion Paper No. 4333.

LÁZARO Y TORRES, M.L. (1990): Precio del suelo y utilización del espacio en la ciudad de Málaga. Madrid: Universidad Complutense de Madrid.

LÁZARO Y TORRES, M. L. (1995): Últimas tendencias en el precio de la vivienda en la ciudad de Málaga (1981-1984). Anales de Geografía de la Universidad Complutense, 15, p. 421-433.

LÁZARO Y TORRES, M. L. y GONZÁLEZ GONZÁLEZ, Ma J. (2009): La inmigración reciente en España: su contribución al crecimiento demográfico y a la economía. Boletín de la Real Sociedad Geográfica, CXLV, p. 183-202.

PAREJA-EASTAWAY, M. (2009): The effects of the Spanish housing system on the settlement patterns of immigrants. Tijdschrift voor economische en sociale geografie, 100, pp. 519-534.

PEREDA, C., ACTIS, W. y PRADA, M.A. de. (2005): Inmigración y vivienda en España. Madrid: MTIN.

PEREDA, C., ACTIS, W. y PRADA, M.A. de. (2006): Dimensiones de la inmigración en España. Impactos y desafíos. Papeles, 103, p. 95-104.

POZO RIVERA, E. y GARCÍA PALOMARES, J.C. (2009): Inmigración y cambio demográfico en la región metropolitana madrileña entre 1996 y 2006. Anales de Geografía de la Universidad Complutense, Vol. 29, n 1, pp. 111-138.

ROMERO, M. (2008): La inmigración va por barrios Diario de León, 27 de noviembre de 2008, p.2.

SAIZ A. (2007) Immigration and housing rents in American cities. Journal of Urban Economics, 61 (2), pp. 345-371.

SOSVILlA, S. (2008): Immigration and Housing Prices in Spain, Documento de trabajo 2008-40, Serie Economía Regional. Fundación de Estudios de Economía Aplicada, Madrid.

STILLMAN, S. y MARÉ, D.C. (2008): Housing Markets and Migration: Evidence from New Zealand, Motu Working Paper 08-06.

ZÁRATE,A. (2002): Manifestaciones de multiculturalidaden el centro de Madrid, disponible en Cervantes Virtual (último acceso 14 de febrero de 2011): http://descargas. cervantesvirtual.com/servlet/SirveObras/12159396447091532976624/020793. pdf?incr $=1$ 